\title{
Asymptotic behavior of the node degrees in the ensemble average of adjacency matrix
}

\author{
YUKIO HAYASHI \\ Japan Advanced Institute of Science and Technology, Ishikawa, Japan \\ (e-mail: yhayashi@jaist.ac.jp)
}

\begin{abstract}
Various important and useful quantities or measures that characterize the topological network structure are usually investigated for a network, then they are averaged over the samples. In this paper, we propose an explicit representation by the beforehand averaged adjacency matrix over samples of growing networks as a new general framework for investigating the characteristic quantities. It is applied to some network models, and shows a good approximation of degree distribution asymptotically. In particular, our approach will be applicable through the numerical calculations instead of intractable theoretical analyses, when the time-course of degree is a monotone increasing function like power law or logarithm.
\end{abstract}

Keywords: ensemble average, growing networks, degree distribution, degree-degree correlations

\section{Introduction}

Many social, technological, and biological networks belong to a common scalefree (SF) structure (Babarási \& Albert, 1999) which consists of many low-degree nodes and a few high-degree nodes called as hubs. The degree distribution follows a power law, therefore an SF network has an extreme vulnerability against hub attacks (Albert et al., 2000). In addition, these real networks are classified into assortative and disassortative networks (Newman, 2003). For examples, typical social networks, e.g. coauthorships and actor collaborations, are assortative, while typical technological and biological networks, e.g. Internet, World-Wide-Web, protein-interaction, and food webs, are disassortative. In assortative networks, nodes with similar degrees tend to be connected, and thus positive degree-degree correlations appear. In disassortative networks, nodes with different degrees: low and high degrees tend to be connected, and thus negative degree-degree correlations appear.

Through the above findings in network science, superior network theories and efficient algorithms have been developed for analyzing network topology and dynamics (Newman, 2010). However, studies for the cases with degree-degree correlations are not clear enough for successful analyses of topological structures and epidemics on networks except some percolation analyses. Recently, it has been numerically and theoretically found that an onion-like structure with positive degreedegree correlations gives the nearly optimal robustness against hub attacks in an SF network (Herrmann et al., 2011; Schneider et al., 2011; Tanizawa et al., 2012).

On the other hand, the average behavior of stochastically generated network models or empirical data samples of real networks is discussed in many applications. 
Usually, some characteristic quantities such as degree distribution or clustering coefficient are investigated for a network, then their quantities are averaged over the samples of networks in which the existence of a generation rule (mechanism) of the networks is assumed. In this paper, we focus on the beforehand averaged network structure over samples, and calculate the degree distribution for several models of growing network with or without degree-degree correlations. This representation will give a general framework for numerically investigating the characteristic topological quantities in growing networks. Since our framework is supported by the interesting property of ordering that older nodes tend to have higher degrees in a randomly growing network (Callaway et al., 2001), a wide range of application to growing networks can be expected.

\section{Representation by the ensemble average of adjacency matrix}

We consider a set of growing networks in which a new node is added with probabilistic links to existing nodes in a network at each time step. To study the average behavior of the stochastic processes in many samples, we use the ensemble average of adjacency matrix defined as follows:

$$
A(t) \stackrel{\text { def }}{=}\left(\begin{array}{ccccccccc}
0 & 1 & a_{13} & \ldots & a_{i j} & \ldots & a_{1 i} & \ldots & a_{1 n} \\
1 & 0 & a_{23} & \ldots & a_{2 j} & \ldots & a_{2 i} & \ldots & a_{2 n} \\
a_{31} & a_{32} & 0 & \ldots & a_{3 j} & \ldots & a_{3 i} & \ldots & a_{3 n} \\
\vdots & \vdots & \vdots & \ddots & \vdots & \vdots & \vdots & \vdots & \vdots \\
a_{j 1} & a_{j 2} & a_{3 j} & \ldots & 0 & \ldots & a_{j i} & \ldots & a_{j n} \\
\vdots & \vdots & \vdots & \vdots & \vdots & \ddots & \vdots & \vdots & \vdots \\
a_{i 1} & a_{i 2} & \ldots & \ldots & a_{i j} & \ldots & 0 & \ldots & a_{i n} \\
\vdots & \vdots & \vdots & \vdots & \vdots & \vdots & \vdots & \ddots & \vdots \\
a_{n 1} & a_{n 2} & \ldots & \ldots & \ldots & \ldots & a_{n i} & \ldots & 0
\end{array}\right)
$$

Here, without loss of generality, we set connected two nodes as the minimum initial configuration: $a_{11}=a_{22}=0, a_{12}=a_{21}=1$, and the degree $k_{1}(0)=k_{2}(0)=1$. Note that at each time step, the matrix is expanding with the inverse $L$ shape of elements $a_{1 n}, a_{2 n}, \ldots, a_{n-1 n}, 0, a_{n n-1}, \ldots, a_{n 2}, a_{n 1}$ at the right-bottom corner. The diagonal element $a_{i i}$ is always 0 due to no self-loop at each node $i$. Other elements are $0 \leqslant a_{i j} \leqslant 1, i \neq j$, as the average number of links from $i$ to $j$ over the samples of networks. The value of each element is defined in order according to the passage of time. We assume that there are no adding links between existing nodes $i, j<n$ at any time $t=n-2$. Only links between selected old nodes and new node $n$ are added in a growing network.

In the sample-based description, the ensemble average of adjacency matrix is

$$
A(t)=\frac{A^{(1)}(t)+A^{(2)}(t)+\cdots+A^{(k)}(t)+\cdots+A^{\left(\mathscr{N}_{s}\right)}(t)}{\mathscr{N}_{s}},
$$

where $A^{(k)}(t)$ denotes an adjacency matrix of the $k$-th sample whose elements are $a_{i j}^{(k)}=1$ or 0 corresponded to the connection or disconnection from $i$ to $j$, but $a_{i j}^{(k)}=0$ or undefined for $i, j>t+2$ because of the size $n=t+2$ at time $t . \mathscr{N}_{s}$ denotes the number of samples. We remark that an adjacency matrix is fundamental 
and important because it includes the necessary and sufficient information about a network structure and is useful for a mathematical treatment.

In this explicit representation of general framework, for each $i$-th node, $i=$ $1,2, \ldots, n-1$, the (out-)degree is updated from time $t-1$ to $t$,

$$
k_{i}(t)=k_{i}(t-1)+a_{i n} .
$$

The (out-)degree of $n$-th node added at time $t=n-2$ is defined by the sum of links from node $n$ to nodes $i$,

$$
k_{n}(t) \stackrel{\text { def }}{=} \sum_{i=1}^{n-1} a_{n i} .
$$

We should remark that the iterative calculations of Equations (1) and (2) are equivalent to the averaged values over the samples after calculating the degrees for each sample of the networks at time $t$. With this equivalence in mind, we investigate the asymptotic behavior of $k_{i}(t)$ for a large $t$. We note that $k_{i}(t)$ is a monotone non-decreasing function of time $t$ because of $k_{i}(t)-k_{i}(t-1)=a_{i n} \geqslant 0$ from Equation (1) in growing networks.

\section{Asymptotic behavior of the node degrees}

As examples, we apply the ensemble average of adjacency matrix to some network models. However, our approach is applicable to other growing networks especially in a wide class, e.g. with approximately power law or exponential degree distribution. In the following, we assume that each link is undirected: $a_{i j}=a_{j i}$.

\subsection{Babarási and Albert model}

Since the continuous-time approximation of Equation (1) is generally

$$
\frac{d k_{i}(t)}{d t}=a_{i n}
$$

our approach is applicable to the Babarási and Albert (BA) model (Babarási et al., 1999) as follows:

Preferential attachment:

$$
a_{n i}=\frac{m \times k_{i}(t-1)}{\sum_{l} k_{l}(t-1)} \approx \frac{k_{i}(t-1)}{2 t},
$$

Uniform attachment:

$$
\forall i \quad a_{n i}=\frac{m}{m_{0}+t-1},
$$

where $m_{0}$ denotes the initial number of nodes, and $m$ denotes the number of adding links at each time step.

For the two cases of preferential and uniform attachments, $k_{i}(t)=m \sqrt{t / t_{i}}$ and $k_{i}(t)=m\left(\log \left(m_{0}+t-1\right)-\log \left(m_{0}+t_{i}-1\right)+1\right)$ have been derived with the corresponding degree distributions $p(k) \sim k^{-3}$ and $p(k) \sim e^{-k}$, respectively (Babarási et al., 1999). The analysis in BA model is based on the invariant ordering property of degrees in which older nodes get more links averagely at any time in the growth of 


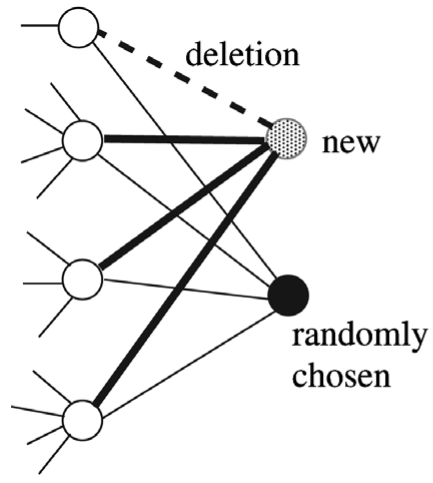

(a) D-D model

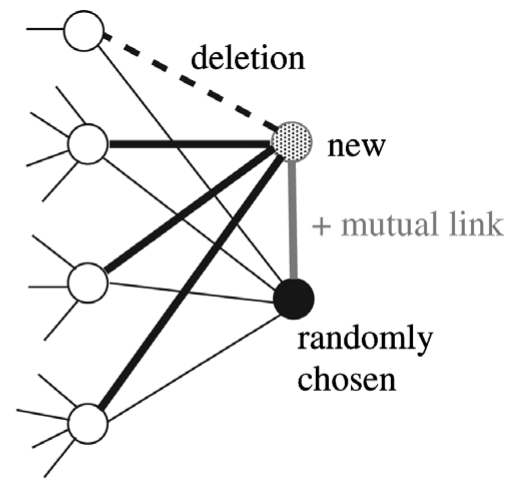

(b) Copying model

Fig. 1. Basic linking processes at each time step from a new node to a randomly chosen node and/or the neighbor nodes in the growing networks. The thick bold lines show adding links, and the dashed line shows a deleted one. The thin lines show already existing links at that time.

network. Under the invariant ordering property, our approach can be regarded as an extension of mathematical treatment in the BA model through the representation by the ensemble average of adjacency matrix over samples of growing networks.

\subsection{Duplication-divergence model}

We preliminary introduce a duplication-divergence (D-D) model (Pastor-Satorras et al., 2003; Sole et al., 2002) without mutations of random links between existing nodes, whose generation mechanism is known as fundamental in biological proteinprotein interaction networks. In the D-D model, at each time step, a new node is added and links to neighbor nodes of a uniformly randomly chosen node (see Figure 1(a)). Some duplication links are deleted with probability $\delta>0$. Here, no mutations are to simplify the discussion and to connect to the next subsection. Although the degree distribution in the D-D model can be approximately analyzed by the approach of mean-field-rate equation (Pastor-Satorras et al., 2003; Sole et al., 2002), we show the applicability of our approach to the D-D model in order to extend it to more general networks. Moreover, in the next section, we reveal that older nodes tend to have higher degrees (Callaway et al., 2001) in the D-D model, whose ordering of degree $k_{i}(t)$ for node index $i$ was not found from the above approach.

Since the $n$-th new node links to the neighbor node $i$ of a chosen node $j \neq i$ from existing nodes $1 \leqslant i, j \leqslant n-1$ in a network of $\mathrm{D}$ - $\mathrm{D}$ model, we have

$$
a_{n i}=\frac{(1-\delta) \sum_{j=1}^{n-1} a_{j i}}{n-1}=\frac{1}{n-1}(1-\delta) k_{i}(t-1),
$$

where we use the uniform selection probability $1 /(n-1)$ of each node $j$ and the no-deletion rate $1-\delta$ for linking to the neighbor nodes.

From Equations (1)-(3), we obtain

$$
k_{i}(t)=\left(1+\frac{1-\delta}{n-1}\right) k_{i}(t-1),
$$




$$
k_{n}(t)=\frac{1-\delta}{n-1} \sum_{i=1}^{n-1} k_{i}(t-1) .
$$

By applying Equation (4) recursively, we derive

$$
\begin{aligned}
k_{i}(t) & =\left(\frac{n-1+(1-\delta)}{n-1}\right)\left(\frac{n-2+(1-\delta)}{n-2}\right) \ldots\left(\frac{i+(1-\delta)}{i}\right) k_{i} \\
& =\frac{\Gamma(n+(1-\delta)) / \Gamma(i+(1-\delta))}{\Gamma(n) / \Gamma(i)} k_{i} \sim(t+1)^{1-\delta} .
\end{aligned}
$$

In addition, the continuous-time approximation of Equation (4) is

$$
\frac{d k_{i}(t)}{d t}=a_{n i}=\frac{1-\delta}{t+1} k_{i}(t)
$$

From the separation of variables method, we obtain the solution

$$
k_{i}(t)=(t+1)^{1-\delta} \sim t^{1-\delta} .
$$

When we denote the initial degree $k_{i}$ at the inserted time $t_{i}$ for a node $i$, the above solution is rewritten as

$$
k_{i}(t) \approx k_{i}\left(\frac{t}{t_{i}}\right)^{1-\delta} .
$$

From the existence of parallel curves shown in Figure 2, the ordering of degrees

$$
k_{n}(t)<k_{n-1}(t)<\cdots<k_{3}(t)<k_{2}(t)=k_{1}(t),
$$

is not changed. In other word, older nodes get more links averagely. Thus, we obtain

$$
\begin{gathered}
p\left(k_{i}(t)<k\right)=p\left(t_{i}>\left(\frac{k_{i}}{k}\right)^{1 /(1-\delta)} t\right)=\left(1-\frac{k_{i}^{1 /(1-\delta)}}{k^{1 /(1-\delta)}}\right) \frac{t}{N_{0}+t}, \\
p(k)=\frac{\partial p\left(k_{i}(t)<k\right)}{\partial k} \sim k^{-\left(1+\frac{1}{1-\delta}\right)},
\end{gathered}
$$

where $N_{0}+t$ is the number of nodes at time $t$, and $N_{0}$ denotes the initial number of nodes. In the tail of degree distribution, the exponent of power law is $1+\frac{1}{1-\delta}$ asymptotically. Note that the slightly different exponent $1+\frac{1}{1-\delta}$ to the conventional approximation (Kim et al., 2002; Pastor-Satorras et al., 2003) is not strange, since the mutations are necessary for their D-D models.

Figure 3(a)-(c) shows the time-course of $k_{i}(t) \sim t^{1-\delta}$ in the case of $\delta=0.3,0.5$, and 0.7 , respectively, averaged over 100 samples. The black, orange, and magenta lines are the numerical results of Equations (4) and (5) for the node $i=1,10$, and 100. The cyan line guides the estimated slope of $1-\delta$ in $\log -\log$ plot. In Figure 3(d), the red, green, and blue lines show the degree distributions for $\delta=0.3,0.5$, and 0.7 , respectively, at the size $n=10^{4}$. The magenta, cyan, and gray dashed lines guides the corresponding slopes of $1+\frac{1}{1-\delta}$ for these $\delta$.

\subsection{Copying model}

A modification (Hayashi, 2014) of the D-D model (Pastor-Satorras et al., 2003; Sole et al., 2002) by adding a mutual link between a new node and a randomly chosen 


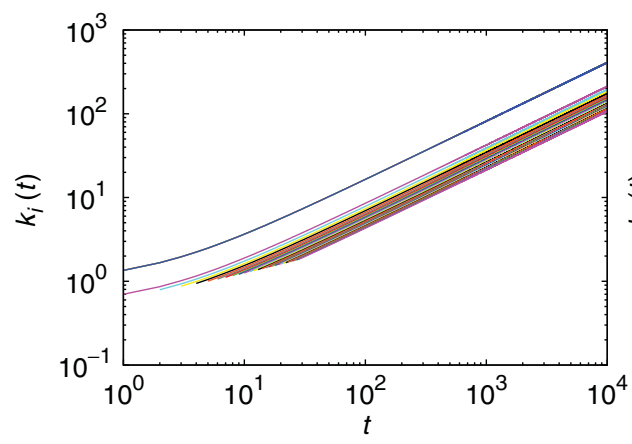

(a) $\delta=0.3$

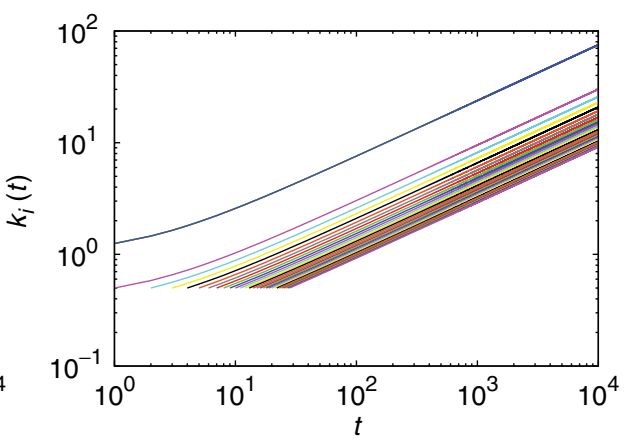

(b) $\delta=0.5$

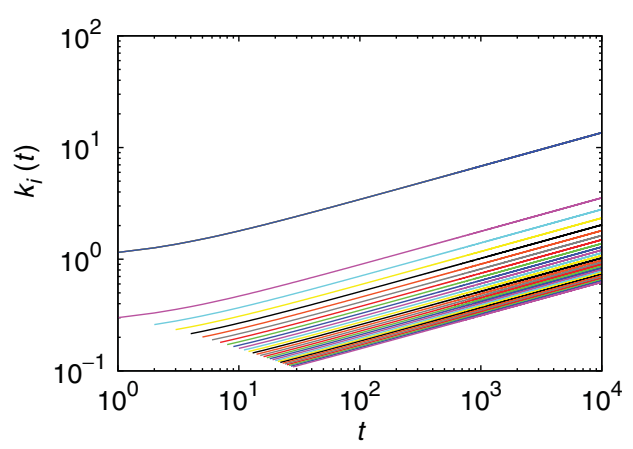

(c) $\delta=0.7$

Fig. 2. Parallel curves of time-course $k_{i}(t)$ of degree in the D-D model with a rate $\delta$ of link deletion. The color lines from top to bottom correspond to the nodes $i=1$ or $2,3,4, \ldots, 30$. Note that each node $i$ is born at time $t_{i}=i-2$. (Color online)

node has been proposed. The mutual link contributes to avoid the singularity called as non-self-averaging even for $\delta<1 / 2$ without mutations (Hayashi, 2014). The growing network is constructed as shown in Figure 1(b). It is referred to as copying model. At each time step, a new node is added. The new node links to a uniformly randomly chosen node, and to its neighbor nodes with probability $1-\delta$.

In the copying model, we have

$$
a_{n i}=\frac{1+(1-\delta) \sum_{j=1}^{n-1} a_{j i}}{n-1}=\frac{1+(1-\delta) k_{i}(t-1)}{n-1},
$$

since the $n$-th new node links to a uniformly randomly chosen node $i$ and to the neighbor node $i$ when other node $j$ is chosen from existing nodes $1 \leqslant i, j \leqslant n-1$ in the network. These effects are in the first term $1 /(n-1)$ and the second term $(1-\delta) \sum_{j=1}^{n-1} a_{j i} /(n-1)$ in Equation (8).

From Equations (1), (2) and (8), we obtain

$$
\begin{gathered}
k_{i}(t)=\frac{1}{n-1}+\left(1+\frac{1-\delta}{n-1}\right) k_{i}(t-1), \\
k_{n}(t)=1+\frac{1-\delta}{n-1} \sum_{i=1}^{n-1} k_{i}(t-1) .
\end{gathered}
$$




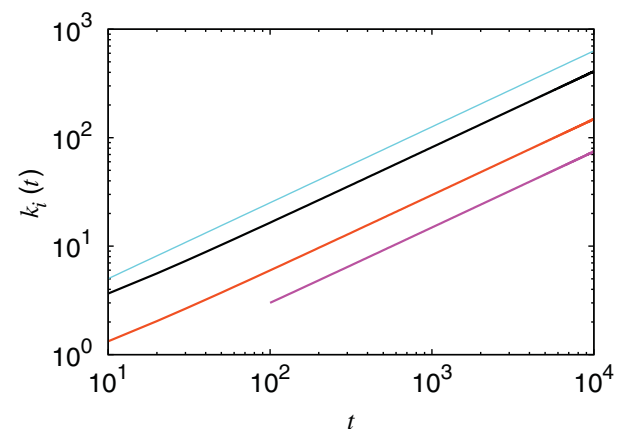

(a) $\delta=0.3$

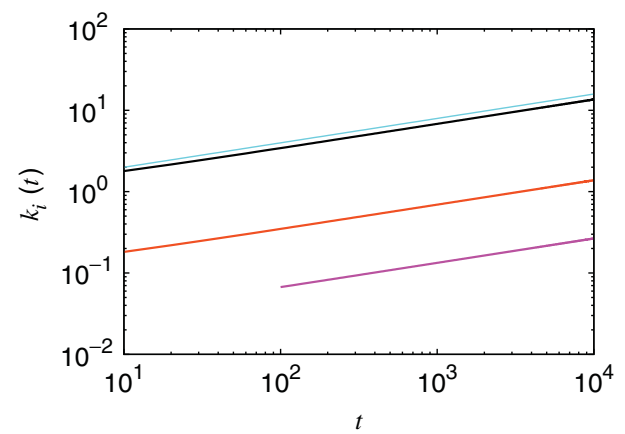

(c) $\delta=0.7$

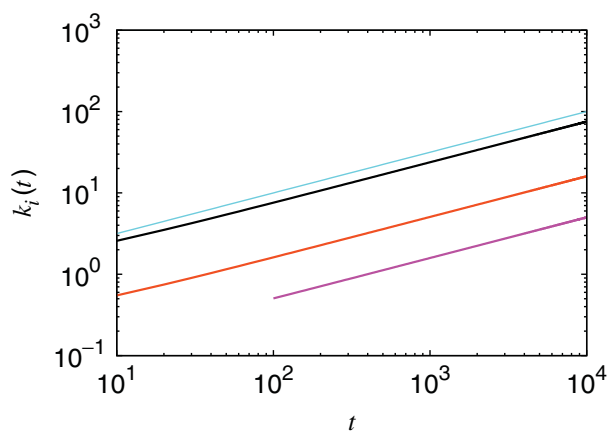

(b) $\delta=0.5$

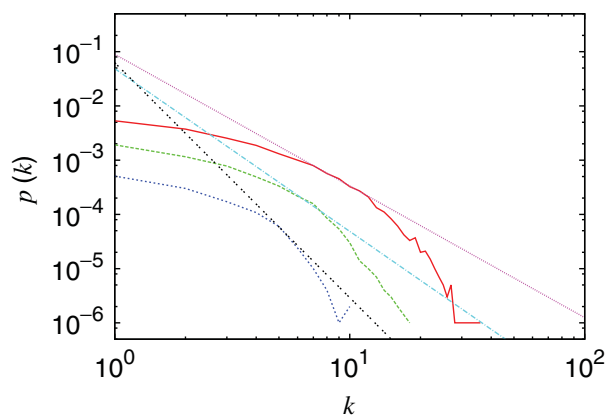

(d) $p(k)$

Fig. 3. Results for the D-D model without mutations. (a)-(c) Time-courses $k_{i}(t)$ of degree for node $i=1,10$, and 100 shown by black, orange, and magenta lines, respectively, at the typical values of rate $\delta$ of link deletion. The cyan line guides the estimated slope $1-\delta$ of $k_{i}(t) \sim t^{1-\delta}$. (d) Degree distribution $p(k)$ in the cases of $\delta=0.3,0.5$, and 0.7 shown by red, green, and blue lines, respectively. The dotted magenta, cyan, and gray lines show the estimated power law distributions $k^{-\left(1+\frac{1}{1-\delta}\right)}$. (Color online)

By applying Equation (9) recursively, we derive

$$
\begin{aligned}
k_{i}(t) & =\frac{1}{t+1}+\left(\frac{t+1+(1-\delta)}{t+1}\right)\left(\frac{1}{t}+\left(\frac{t+(1-\delta)}{t} k_{i}(t-2)\right)\right) \\
& =\frac{1}{t+1}+\frac{t+1+(1-\delta)}{(t+1) t}+\cdots+\frac{\Gamma(t+2+(1-\delta)) / \Gamma(i)}{\Gamma(t+2) / \Gamma(i)} k_{i}\left(t_{i}\right) \\
& \approx O(1 / t)+(t+1)^{1-\delta}
\end{aligned}
$$

where we use $n-1=t+1$ and the Staring formula $\Gamma(x+1) \approx x^{x} e^{-x}$.

In particular, by the mathematical induction, we confirm that the case of $\delta=0$ generates a sequence of the complete graphs with $k_{i}(t)=t+1=n-1$ links at every node of $i=1,2, \ldots, n$. First, $k_{1}(1)=k_{2}(1)=2$ and $k_{3}(1)=2$ are obvious. Next, we assume $k_{i}(t-1)=(t-1)+1=n-2$, from Equations (9) and (10), we derive

$$
\begin{aligned}
k_{i}(t) & =\frac{1}{n-1}+\left(1+\frac{1}{n-1}\right)(n-2)=\frac{1+(n-1)(n-2)+(n-2)}{n-1} \\
& =\frac{(n-1)^{2}}{n-1}=n-1
\end{aligned}
$$




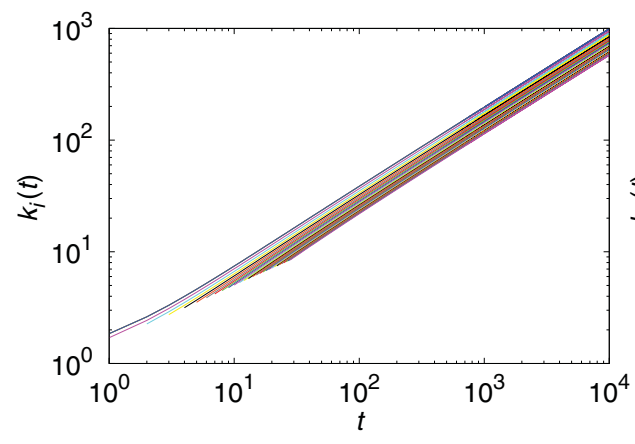

(a) $\delta=0.3$

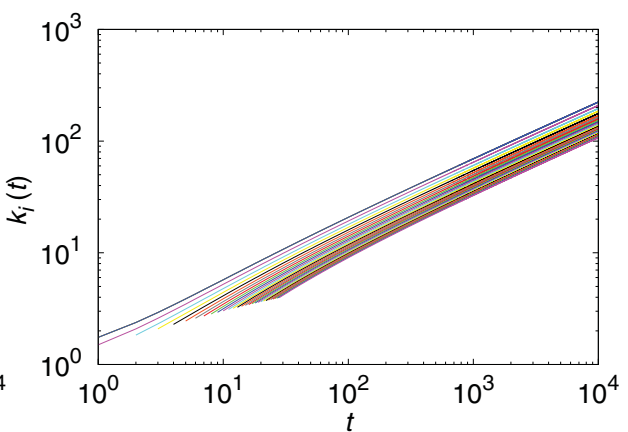

(b) $\delta=0.5$

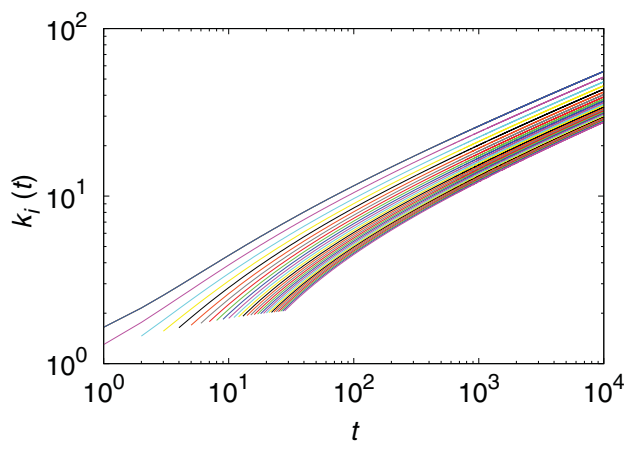

(c) $\delta=0.7$

Fig. 4. Parallel curves of time-course $k_{i}(t)$ of degree in the copying model with a rate $\delta$ of link deletion. The color lines from top to bottom correspond to the nodes $i=1$ or $2,3,4, \ldots, 30$. Note that each node $i$ is born at time $t_{i}=i-2$. (Color online)

$$
k_{n}(t)=1+\frac{(n-1)(n-2)}{n-1}=n-1 .
$$

On the other hand, the continuous-time approximation of Equation (9) is

$$
\frac{d k_{i}(t)}{d t}=a_{n i}=\frac{1}{t+1}+\left(\frac{1-\delta}{t+1}\right) k_{i}(t) .
$$

Since this form is a first-order linear differential equation $d k_{i} / d t+f(t) k_{i}=g(t)$, by applying the solution $e^{-\int f(t) d t}\left(e^{\int f(t) d t} g(t) d t+A\right)$, we obtain

$$
k_{i}(t)=(t+1)^{1-\delta}\left(\int_{0}^{t} \frac{1}{(t+1)^{1+(1-\delta)}} d t+A\right)=A(t+1)^{1-\delta}-C^{\prime} \sim t^{1-\delta},
$$

where $A$ and $C^{\prime}$ are constants of integration, and we use $f(t) \stackrel{\text { def }}{=}-(1-\delta) /(t+1)$ and $g(t) \stackrel{\text { def }}{=} 1 /(t+1)$. Note that the solution $t^{1-\delta}$ is only different by $O(1 / t)$ to Equation (11), and can be ignored for a large $t$. As similar to the D-D model in subsection 3.2, from Equation (7) under the invariant ordering (6) in the parallel curves shown as Figure 4, the degree distribution asymptotically follows a power law with the exponent $1+\frac{1}{1-\delta}$.

Figure 5(a)-(c) shows the time-course of $k_{i}(t) \sim t^{1-\delta}$ in the cases of $\delta=0.3,0.5$, and 0.7 , respectively, averaged over 100 samples. The black, orange, and magenta lines are the numerical results of Equations (9) and (10) for the node $i=1,10$, and 


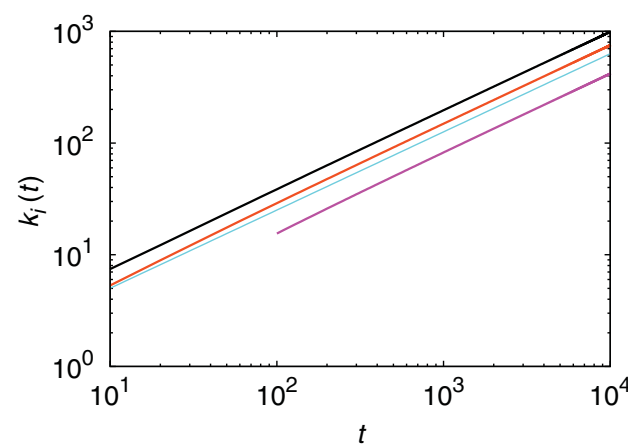

(a) $\delta=0.3$

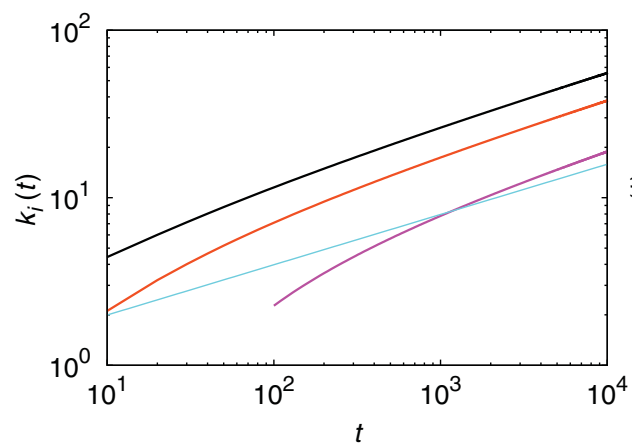

(c) $\delta=0.7$

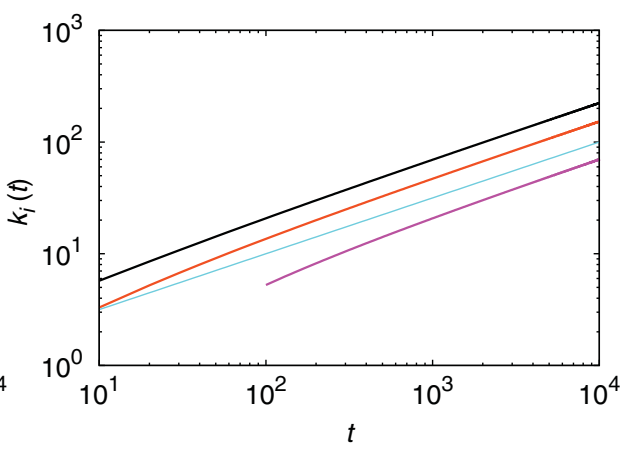

(b) $\delta=0.5$

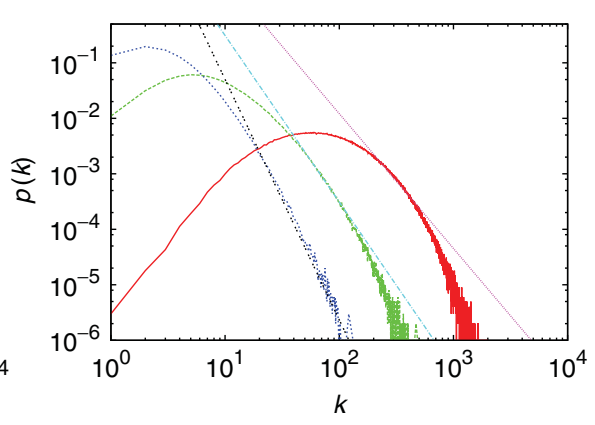

(d) $p(k)$

Fig. 5. Results for the copying model. (a)-(c) Time-courses $k_{i}(t)$ of degree for node $i=1,10$, and 100 shown by black, orange, and magenta lines, respectively, at the typical values of rate $\delta$ of link deletion. The cyan line guides the estimated slope $1-\delta$ of $k_{i}(t) \sim t^{1-\delta}$. (d) Degree distribution $p(k)$ in the cases of $\delta=0.3,0.5$, and 0.7 shown by red, green, and blue lines, respectively. The dotted magenta, cyan, and gray lines show the estimated power-law distributions $k^{-\left(1+\frac{1}{1-\delta}\right)}$. (Color online)

100. The cyan line guides the estimated slope of $1-\delta$ in $\log -\log$ plot. It fits to the lines of $k_{i}(t)$ for a large $t$. Moreover, as shown in Figure 5(d), Equation (7) gives a good approximation at the size $n=10^{4}$. The red, green, and blue lines show the degree distributions for $\delta=0.3,0.5$, and 0.7 , respectively. The magenta, cyan, and gray dashed lines guides the corresponding slopes of $1+\frac{1}{1-\delta}$ for these $\delta$ in the fitting to the tails.

\subsection{Copying model with positive degree-degree correlations}

In this subsection, we emphasize that our approach is effective through the numerical estimation, even when an analytic derivation is intractable.

We consider a copying model with positive degree-degree correlations based on a cooperative generation mechanism by linking homophily, in which densely connected cores among high-degree nodes emerge (Hayashi, 2014). In more detail, the difference to the previously mentioned copying model is that the $n$-th new node links to the neighbor nodes $j$ of a randomly chosen node $i$ with a probability 


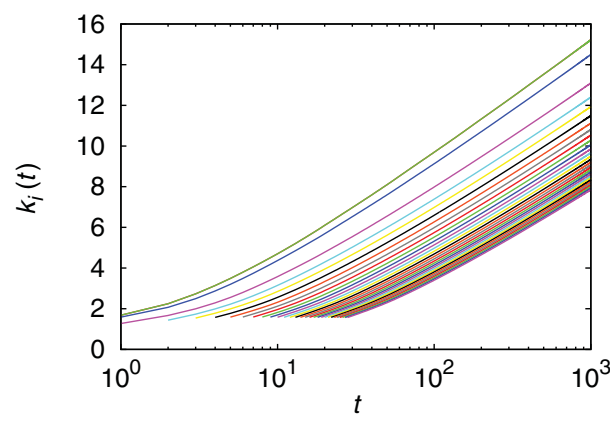

(a) $\delta=0.3$

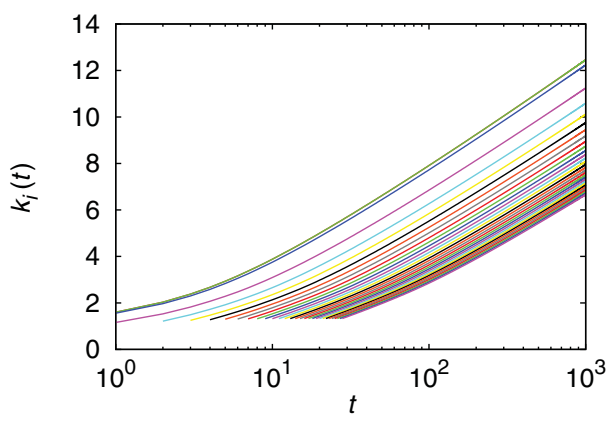

(b) $\delta=0.5$

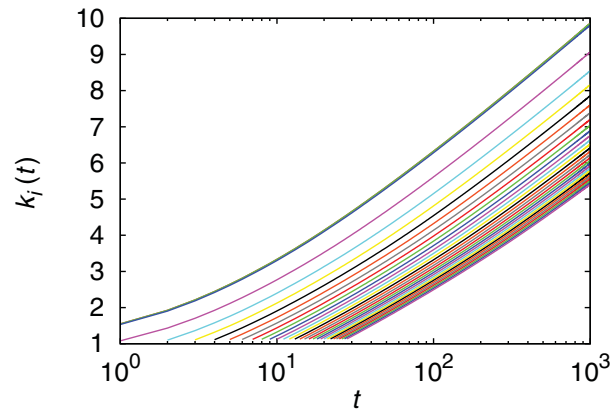

(c) $\delta=0.7$

Fig. 6. Parallel curves of time-course $k_{i}(t)$ of degree in the copying model with positive degree-degree correlations and a rate $\delta$ of link deletion. The color lines from top to bottom correspond to the nodes $i=1$ or $2,3,4, \ldots, 30$. Note that each node $i$ is born at time $t_{i}=i-2$. (Color online)

$(1-\delta) f\left((1-\delta) k_{i}(t-1), k_{j}(t-1)\right)$ from existing nodes in the network. Such a function

$$
f(x, y) \stackrel{\text { def }}{=} \frac{1}{1+a|x-y|},
$$

is necessary to enhance the degree-degree correlations, and $a>0$ is a parameter (Wu \& Holme, 2011). Since the degree of new node is unknown in advance due to the stochastic process, it is temporary set as $(1-\delta) k_{i}(t-1)$.

Thus, instead of Equation (8), we substitute

$$
a_{n i} \stackrel{\text { def }}{=} \frac{1+(1-\delta) \sum_{j=1}^{n-1} a_{j i} \times f\left((1-\delta) k_{i}(t-1), k_{j}(t-1)\right)}{n-1},
$$

for Equations (1) and (2).

Although the theoretical analysis of Equations (1) and (2) for Equation (12) is difficult, the iterative calculations are possible numerically. When we assume $\beta k_{i}(t) \sim \log (t)$, we derive an exponential distribution as follows. From $t \sim e^{\beta k_{i}(t)}$, we obtain

$$
\begin{gathered}
\frac{t_{i}}{t}=\frac{e^{\beta k_{i}}}{e^{\beta k_{i}(t)}}, \\
p\left(k_{i}(t)<k\right)=p\left(t_{i}>\frac{e^{\beta k_{i}}}{e^{\beta k}} t\right)=\left(1-\frac{e^{\beta k_{i}}}{e^{\beta k}}\right) \frac{t}{N_{0}+t},
\end{gathered}
$$




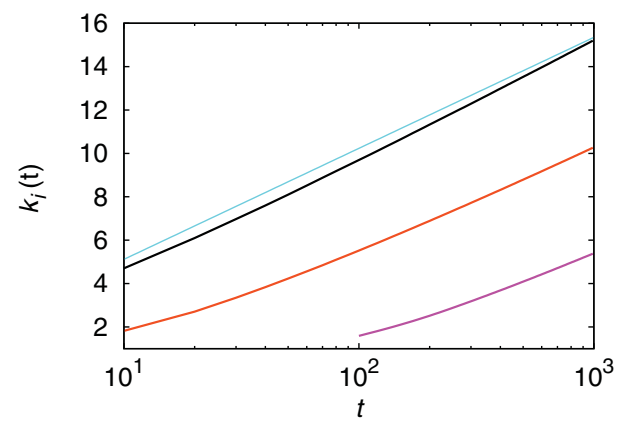

(a) $\delta=0.3$

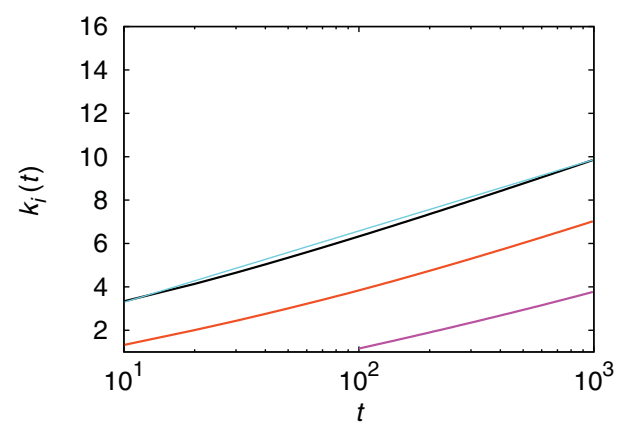

(c) $\delta=0.7$

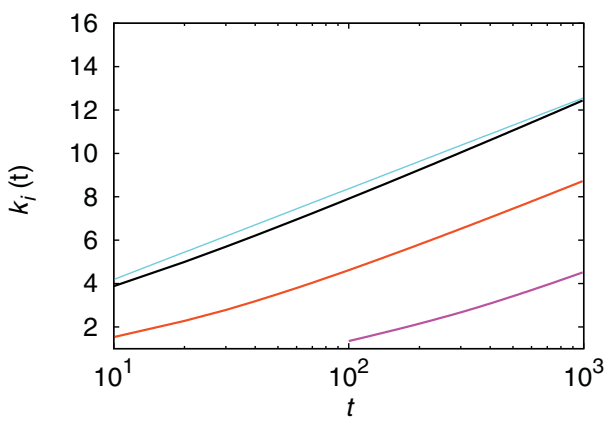

(b) $\delta=0.5$

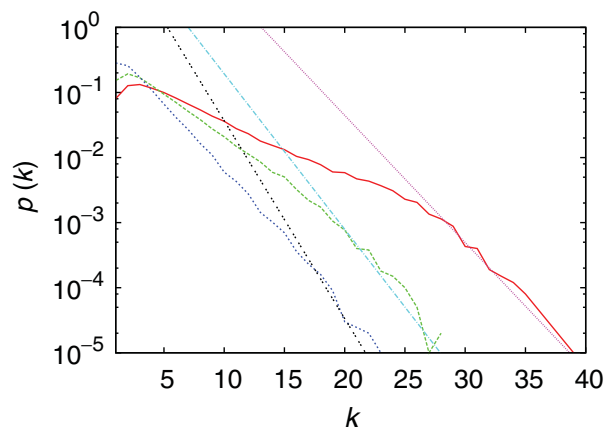

(d) $p(k)$

Fig. 7. Results for the copying model with positive degree-degree correlations. (a)-(c) Timecourses $k_{i}(t)$ of degree for node $i=1,10$, and 100 shown by black, orange, and magenta lines, respectively, at the typical values of rate $\delta$ of link deletion. The cyan line guides the estimated slope $1 / \beta$ of $k_{i}(t) \sim \log (t) / \beta$ in semi-log plot. (d) Degree distribution $p(k)$ in the cases of $\delta=0.3,0.5$, and 0.7 shown by red, green, and blue lines, respectively. The dotted magenta, cyan, and gray lines show the estimated exponential distributions $e^{-\beta k}$. (Color online)

$$
p(k)=\frac{\partial p\left(k_{i}(t)<k\right)}{\partial k} \sim e^{-\beta k}
$$

under the invariant ordering (6) in the parallel curves shown as Figure 6.

Figure $7(\mathrm{a})-(\mathrm{c})$ shows that $k_{i}(t)$ denoted by black, orange, and magenta lines for $i=1,10$, and 100 is approximated by $\log (t)$ in the copying model with degreedegree correlations. The cyan lines guide the estimated slopes $\beta=2.222,1.818$, and 1.428 for $\delta=0.3,0.5$, and 0.7 , respectively, in the numerical fittings for the iterative calculations of Equations (1), (2) and (12). Thus, as shown in Figure 7(d), the tails in $p(k)$ denoted by red, green, and blue lines for $\delta=0.3,0.5$, and 0.7 are approximated by $e^{-\beta k}$ shown as magenta, cyan, and gray dashed lines at the size $n=10^{3}$. Note that $p(k)$ is only slightly deviated but the exponential part is remained by adding shortcut links in order to self-organize a robust onion-like structure in the incrementally growing network (Hayashi, 2014).

\subsection{More general case}

We consider the asymptotic behavior of the node degrees for a general case in growing networks. When the time-course $k_{i}(t)$ of degree follows a monotone increasing function $g(t)$ of time $t$, there exists the inverse function $h(k) \stackrel{\text { def }}{=} g^{-1}(k)=t$. 
It is possible that the time-course $k_{i}(t)$ is an average of observed real data for node $i$, e.g. born at time $t-2$. From

we have

$$
\frac{t_{i}}{t}=\frac{h\left(k_{i}\right)}{h(k)},
$$

$$
p\left(k_{i}(t)<k\right)=p\left(t_{i}>\frac{h\left(k_{i}\right)}{h(k)} t\right) .
$$

Then, on the assumption of the invariant ordering (6) in parallel curves of $\left\{k_{i}(t)\right\}$, we derive

$$
p(k)=\frac{\partial p\left(k_{i}(t)<k\right)}{\partial k}=\frac{\partial}{\partial k}\left(1-\frac{\text { const. }}{h(k)}\right) \frac{t}{N_{0}+t} \sim \frac{h^{\prime}(k)}{h^{2}(k)},
$$

where $h^{\prime}(k)$ denote the derivative of $h(k)$ by the variable $k$. We should remark that various degree distributions of non-power law may appear depending on the shape of monotone increasing function $k_{i}(t)$ according to what type of generation in growing networks.

\section{Analytic deviation for the invariant ordering of node degrees}

We derive the invariant ordering (6) at any time $t$ within parallel curves of monotone increasing functions for the D-D and copying models discussed in subsections 3.2 and 3.3. In the following, we use double mathematical induction for node index $i$ and time $t$.

Once $k_{i}(t-1)-k_{j}(t-1)>0$ is satisfied for 1 or $2 \leqslant i<j \leqslant n-1$ at $t=n-2 \geqslant 1$,

$$
\begin{aligned}
k_{i}(t)-k_{j}(t) & =\left(k_{i}(t-1)-k_{j}(t-1)\right)+\left(a_{i n}-a_{j n}\right) \\
& =\left(1+\frac{1-\delta}{n-1}\right)\left(k_{i}(t-1)-k_{j}(t-1)\right)>0,
\end{aligned}
$$

is obtained from Equations (1), (4) and (9). Here, from Equations (4) and (5) at $n=3$ with the initial condition $k_{1}(0)=k_{2}(0)=1$, we obtain

$$
k_{1}(1)=k_{2}(1)=1+\frac{1-\delta}{2}, \quad k_{3}(1)=1-\delta<k_{1}(1)=k_{2}(1) .
$$

From Equations (9) and (10) at $n=3$ with the same initial condition, we also obtain

$$
k_{1}(1)=k_{2}(1)=2-\frac{\delta}{2}, \quad k_{3}(1)=1+\frac{1-\delta}{2}(1+1)<k_{1}(1)=k_{2}(1) .
$$

Since the assumption in Equation (13) is satisfied for $i=1$ or 2 and $j=3$ at $t=2$, we have

$$
\begin{aligned}
k_{3}(2) & <k_{2}(2)=k_{1}(2), \\
& \vdots \\
k_{3}(t) & <k_{2}(t)=k_{1}(t),
\end{aligned}
$$

by applying Equation (13) recursively for $t \geqslant 2$.

On the other hand, from Equations (4) and (5), we rewrite

$$
k_{n}(t)=\frac{1-\delta}{n-1}\left(\sum_{i=1}^{n-2} k_{i}(t-1)+k_{n-1}(t-1)\right) \text {, }
$$




$$
\begin{gathered}
k_{n-1}(t)=\left(1+\frac{1-\delta}{n-1}\right) k_{n-1}(t-1) \\
k_{n-1}(t-1)=\frac{1-\delta}{n-2} \sum_{i=1}^{n-2} k_{i}(t-2),
\end{gathered}
$$

at $t=n-2$, and

$$
k_{i}(t-1)=\left(1+\frac{1-\delta}{n-2}\right) k_{i}(t-2),
$$

for $i=1,2, \ldots, n-2$. Then, we have

$$
k_{n-1}(t)-k_{n}(t)=\frac{1-\delta}{n-2}\left(1-\frac{n-1-\delta}{n-1}\right) \sum_{i=1}^{n-2} k_{i}(t-2)>0 .
$$

Similarly, from Equations (9) and (10), we rewrite

$$
\begin{gathered}
k_{n}(t)=1+\frac{1-\delta}{n-1}\left(\sum_{i=1}^{n-2} k_{i}(t-1)+k_{n-1}(t-1)\right), \\
k_{n-1}(t)=\frac{1}{n-1}+\left(1+\frac{1-\delta}{n-1}\right) k_{n-1}(t-1), \\
k_{n-1}(t-1)=1+\frac{1-\delta}{n-2} \sum_{i=1}^{n-2} k_{i}(t-2),
\end{gathered}
$$

at $t=n-2$, and

$$
k_{i}(t-1)=\frac{1}{n-2}+\left(1+\frac{1-\delta}{n-2}\right) k_{i}(t-2),
$$

for $i=1,2, \ldots, n-2$. Then, we also have

$$
k_{n-1}(t)-k_{n}(t)=\frac{\delta}{n-1}+\frac{1-\delta}{n-2}\left(1-\frac{n-1-\delta}{n-1}\right) \sum_{i=1}^{n-2} k_{i}(t-2)>0 .
$$

By applying Equation (13) recursively for $t>n-2$ after substituting Equation (15) or (16) to the right-hand side of Equation (13), we obtain

$$
\begin{aligned}
k_{3}(t)-k_{4}(t) & >0, \\
k_{4}(t)-k_{5}(t) & >0, \\
\vdots & \\
k_{n-1}(t)-k_{n}(t) & >0 .
\end{aligned}
$$

From Equations (14) and (17), we obtain the ordering (6) after all.

Next, we consider the existing condition of the ordering (6) within parallel curves of $\left\{k_{i}(t)\right\}$ for a general case of growing networks discussed in subsection 3.5.

From Equation (1) for 1 or $2 \leqslant i<j \leqslant n-1$ at $t=n-2 \geqslant 2$, we have

$$
k_{i}(t)-k_{j}(t)=\left(k_{i}(t-1)-k_{j}(t-1)\right)+\left(a_{i n}-a_{j n}\right) .
$$

If older nodes tend to get more links,

$$
a_{i n}>a_{j n}
$$


hold in the ensemble average of adjacency matrix. Then, we remark $k_{3}(t)<k_{2}(t)=$ $k_{1}(t)$ from Equation (19) and $k_{3}(1)=k_{3}(0)+a_{13}+a_{23}<k_{1}(1)=k_{1}(0)+a_{31}$ or $k_{2}(1)=k_{2}(0)+a_{32}$ with the initial values $k_{3}(0)=0$ and $k_{1}(0)=k_{2}(0)=1$.

On the other hand, from Equations (1) and (2), we derive

$$
k_{n-1}(t)-k_{n}(t)=\sum_{l=1}^{n-2}\left(a_{(n-1) l}-a_{n l}\right) .
$$

If Equation (19) hold with $a_{i j}=a_{j i}$, for $n \geqslant 4$, we have

$$
k_{n-1}(t)-k_{n}(t)>0 \text {. }
$$

Therefore, on the condition (19), we obtain the ordering (6) from Equations (18) and (20).

\section{Conclusion}

We have proposed the explicit representation by the ensemble average of adjacency matrix over samples of growing networks. The important point is that the adjacency matrix is averaged in advance before calculating a characteristic quantity about the topological structure for each sample. The ensemble average has been applied to some network models: BA (Babarási et al., 1999), D-D (Pastor-Satorras et al., 2003; Sole et al., 2002), and copying (Hayashi, 2014) models for investigating the degree distributions in the asymptotic behavior by using the theoretical and numerical analyses for difference equations and the corresponding continuous-time approximation of differential equations with variables $t$ and $k_{i}(t)$. We have derived $k_{i}(t) \sim t^{1-\delta}$ and $p(k) \sim k^{-\left(1+\frac{1}{1-\delta}\right)}$ for the D-D and copying models under the invariant ordering of degrees which is supported in randomly grown networks (Callaway et al., 2001). Moreover, for the copying model with positive degree-degree correlations, we have shown that the numerical calculations of the difference equation give a good approximation of an estimated exponential distribution, even when an analytic derivation is intractable. The copying model with positive degree-degree correlations is related to the self-organization (Hayashi, 2014) of robust onion-like networks (Herrmann et al., 2011; Schneider et al., 2011; Tanizawa et al., 2012).

Our approach may be also applicable to data analysis for social networks, when the observed time-course of degree is a monotone increasing function like power law or logarithm in the average over samples by ignoring short-time fluctuations, and a node index $i$ or $j$ represents an ordering of its birth time. This expectation is supported as follows. It is helpful for grasping a trend to study the average behavior of many users (nodes) added at a same (sampling interval) time into a network community. Random growth in a social network probably corresponds to encountered chances among people. Moreover, as time goes by, the number of his/her friends for a member of social networks is usually increasing. It is natural that the connections to friends are maintained. However, we must consider the effect of rewirings between old members in the definition of adjacency matrix. Also from a practical viewpoint, $O\left(n^{2}\right)$ space to store an adjacency matrix may cause a problem for dig data.

From the conventional analysis for special network models to a general framework, the representation by the ensemble average will open a door for investigating the 
characteristic quantities, e.g. node degrees in growing networks. In particular, the time-course of a quantity depending on the birth time of node is considered as a key point. The discussion about other quantities such as clustering coefficient or the number of paths of a given length requires further studies of how to analyze the average behavior related to the transitivity.

\section{Acknowledgments}

The author would like to thank anonymous reviewers for their valuable comments. This research is supported in part by a Grant-in-Aid for Scientific Research in Japan, No. JP 25330100.

\section{References}

Albert, R., Jeong, H., \& Babarási, A.-L. (2000). Error and attack tolerance of complex networks. Nature, 406(6794), 378-382.

Babarási, A.-L., \& Albert, R. (1999). Emergence of scaling in random networks. Science, 286(5439), 509-512.

Babarási, A.-L., Albert, R., \& Jeong, H. (1999). Mean-field theory for scale-free random networks. Physica A, 272(1-2), 173-187.

Callaway, D. S., Hopcroft, J. E., Kleinberg, J. M., Newman, M. E. J., \& Strogatz, S. H. (2001). Are randomly grown graphs really random?. Physical Review E, 64(4), 041902.

Hayashi, Y. (2014). Growing self-organized design of efficient and robust complex networks. Proc. of 2014 IEEE 8th Int. Conf. on SASO: SelfAdaptive and Self-Organizing Systems, pp. 50-59. IEEE Xplore Digital Library, http://ieeexplore.ieee.org/xpl/articleDetails.jsp?arnumber=7001000, http://arxiv.org/abs/ 1411.7719

Herrmann, H. J., Schneider, C. M., Moreira, A. A., Andrade Jr. J. S., \& Havlin, S. (2011). Onion-like network topology enhances robustness against malicious attacks. Journal of Statistical Mechanics, Issue 01 P01027, doi: 10.1088/1742-5468/2011/01/P01027.

Kim, J., Krapivsky, P. L., \& Redner, S. (2002). Infinite-order percolation and giant fluctuations in a protein interaction networks. Physical Review E, 66(5), 055101(R).

Newman, M. E. J. (2003). Assortative mixing in networks. Physical Review Letters 89(20), 208701.

Newman, M. E. J. (2010). Networks - an introduction. Oxford University Press, New York.

Pastor-Satorras, R., Smith, E., \& Sole, R. V. (2003). Evolving protein interaction networks through gene duplication. Journal of Theoretical Biology, 222(2), 199-210.

Schneider, C. M., Moreira, A. A., Andrade Jr. J. S., Havlin, S., \& Herrmann, H. J. (2011). Mitigation of malicious attacks on networks. Proceedings of the National Academy of Sciences of the United States of America, 108(10), 3838-3841.

Sole, R. V., Pastor-Satorras, R., Smith, E., \& Kepler, T. B. (2002). A model of large-scale proteome evolution. Advances in Complex Systems, 5(1), 43-54.

Tanizawa, T., Havlin, S., \& Stanley, H. E. (2012). Robustness of onionlike correlated networks against targeted attacks. Physical Review E, 85(4), 046109.

Wu, Z.-X., \& Holme, P. (2011). Onion structure and network robustness. Physical Review E, 84(2), 026106. 